\title{
High-z radio starbursts host X-ray AGN
}

\author{
Anita M. S. Richards ${ }^{1}$, R. Beswick ${ }^{1}$, S. T. Garrington ${ }^{1}$, T. W. B. \\ Muxlow ${ }^{1}$, H. Thrall ${ }^{1}$, M. A. Garrett ${ }^{2}$, M. Kettenis ${ }^{2}$, H. J. van \\ Langevelde $^{2}$, E. Gonzalez-Solarez ${ }^{3}$, N. A. Walton ${ }^{3}$ and M. G. Allen \\ ${ }^{1}$ Jodrell Bank Observatory, University of Manchester, SK11 9DL, UK. amsr@jb.man.ac.uk \\ ${ }^{2}$ Joint Institute for VLBI in Europe, Postbus 2, 7990 AA Dwingeloo, The Netherlands \\ ${ }^{3}$ Institute of Astronomy, Madingley Road, Cambridge CB3 0HA, UK. \\ ${ }^{4}$ CDS (UMR 7550), 11 rue de l'Université, 67000 Strasbourg, France.
}

\begin{abstract}
We use Virtual Observatory methods to investigate the association between radio and X-ray emission at high redshifts. Fifty-five of the $92 \mathrm{HDF}(\mathrm{N})$ sources resolved by combining MERLIN+VLA data were detected by Chandra, of which 18 are hard enough and bright enough to be obscured AGN. The high- $z$ population of $\mu \mathrm{Jy}$ radio sources is dominated by starbursts an order of magnitude more active and more extended than any found at $z<1$ and at least a quarter of these simultaneously host highly X-ray-luminous obscured AGN.
\end{abstract}

Keywords. galaxies: starburst, galaxies: active, radio continuum: galaxies, X-rays: galaxies, techniques: high angular resolution, surveys

A region of $100 \operatorname{arcmin}^{2}$ around the Hubble Deep Field North was observed by the MERLIN and the VLA (Muxlow et al. 2005). 92 sources brighter than $40 \mu \mathrm{Jy}$ were detected at $1.4 \mathrm{GHz}$ and all showed structure at $0 .{ }^{\prime \prime} 2-2^{\prime \prime}$ resolution. These are the only observations apart from the HST images which provide morphological information. We were thus able to classify the specific origins of the radio emission in each galaxy, which may be different from the sources of optical and other radiation from the same object. We used the presence of rest-frame FIR emission (e.g. Garrett 2002) and the HST images (Giavalisco et al. 2004) to provide supporting information only. Compact bright peaks with a flat radio spectrum are probably AGN whilst extended emission with a steep non-thermal spectrum is likely to be of starburst origin. The latter are often associated with ISO, Spitzer or SCUBA sources (e.g. Chapman et al. 2005) and with interacting or distorted galaxies in the HST images. 55 of the radio sources are among the $100 \mathrm{X}$ ray sources detected by Chandra in the same field (Alexander et al. 2003), including 18 obscured AGN identified by Padovani et al. 2004 (with X-ray luminosities $>10^{35} \mathrm{~W}$ and photon indices $\geqslant 1$ ) but the radio emission is starburst dominated in at least 11 of these. The mean angular size of the radio sources in the HDFN is $1^{\prime \prime} .3$, corresponding to a typical extent of 8-10 kpc for starbursts and the inferred star-formation rates are 1-2000 $\mathrm{M}_{\odot} \mathrm{yr}^{-1}$ for the higher-redshift sources. This is an order of magnitude more exended and more vigourous than for local ULIRGS.

\section{References}

Alexander, D. M., et al., 2003, AJ, 126, 539

Chapman, S. C., Blain, A. W., Smail, I., \& Ivison, R. J., 2005, ApJ, 622, 772

Garrett, M. A., 2002, A\&A, 384, L19

Giavalisco, M. et al., 2004, ApJ, 600, L93

Muxlow, T. W. B., et al., 2005, MNRAS, 358, 1159

Padovani, P., Allen, M. G., Rosati, P., \& Walton, N. A., 2004, A\&A, 424, 545 